\title{
BMJ Open A cross-sectional study using freedom of information requests to evaluate variation in local authority commissioning of community pharmacy public health services in England
}

\author{
Adam John Mackridge, ${ }^{1}$ Nicola Jane Gray, ${ }^{2}$ Janet Krska ${ }^{3}$
}

To cite: Mackridge AJ, Gray NJ, Krska J. A cross-sectional study using freedom of information requests to evaluate variation in local authority commissioning of community pharmacy public health services in England. BMJ Open 2017;7:e015511. doi:10.1136/ bmjopen-2016-015511

- Prepublication history for this paper is available online. To view these files, please visit the journal online (http://dx.doi org/10.1136/bmjopen-2016015511).

Received 9 December 2016 Revised 9 May 2017 Accepted 19 May 2017

CrossMark

${ }^{1}$ School of Pharmacy and Biomolecular Sciences, Liverpool John Moores University, Liverpool, UK ${ }^{2}$ Green Line Consulting Limited, Manchester, UK

${ }^{3}$ Medway School of Pharmacy, Universities of Greenwich and Kent, Chatham Maritime, UK

Correspondence to

Professor Janet Krska; j.krska@ kent.ac.uk

\section{ABSTRACT}

Objectives This study aims to provide a national picture of the extent and nature of public health services commissioned by local authorities (LAs) from community pharmacies across England in financial year 2014/15.

Design Cross-sectional survey of public health services commissioned in community pharmacies by LAs, gathered via freedom of information requests and documentary analysis.

Setting and participants All 152 LAs in England. Results A total of 833 commissioned services were reported across England (range 3-10 per LA). Four services were commissioned by over $90 \%$ of LAs: emergency hormonal contraception (EHC), smoking cessation support, supervised consumption of methadone or other opiates and needle and syringe programmes (NSPs). The proportion of pharmacies commissioned to deliver these services varied considerably between LAs from $<10 \%$ to $100 \%$. This variation was not related to differences in relevant proxy measures of need. NHS Health Checks and alcohol screening and brief advice were commissioned by fewer LAs (32\% and $15 \%$, respectively), again with no relationship to relevant measures of need. A range of other services were commissioned less frequently, by fewer than $10 \%$ of LAs. Supervised consumption and NSPs were the most frequently used services, with over 4.4 million individual supervisions and over 1.4 million needle packs supplied. Pharmacies provided over 200000 consultations for supply of EHC, over 30000 supplies of free condoms and almost 16000 chlamydia screening kits. More than 55000 people registered to stop smoking in a community pharmacy, almost 30000 were screened for alcohol use and over 26000 NHS Health Checks were delivered.

Conclusions There is significant variation in commissioning and delivery of public health services in community pharmacies across England, which correlate poorly with potential benefit to local populations. Research to ascertain reasons for this variation is needed to ensure that future commissioning and delivery of these services matches local need.
Strengths and limitations of this study

- The study covered all local authorities (LAs) in England which commissioned community pharmacy public health services in 2014/15.

- Multiple sources were used to minimise missing data; hence, the number of services is complete, numbers of pharmacies providing services is $90 \%$ complete and usage data is $58 \%$ complete.

- The findings rely on the accuracy of the data supplied by LAs, which could not be verified.

- Relevant measures of population health were used to assess potential need for individual services across LA areas, but are recognised as a proxy measure.

- The study did not take account of similar service provision by other agencies.

\section{INTRODUCTION}

National Government in England, community pharmacy contractors' representatives and pharmacy professional bodies have repeatedly expressed the desire for community pharmacy to deliver public health interventions to help promote health and well-being in a wider population. ${ }^{1-5}$ Community pharmacy's particular strengths in providing such services have been suggested as follows: widening access to services, increasing patient's choice and the potential to reach underserved groups. ${ }^{5}$ Indeed, community pharmacies are accessible within a 20-min walk for almost $90 \%$ of the English population, with this rising to almost $100 \%$ for those areas in the most deprived decile. ${ }^{6}$ Public health services are commissioned from community pharmacies through multiple mechanisms, including under the English Community Pharmacy Contractual Framework, local pharmaceutical services contracts and commissioning through local authorities (LAs) and clinical commissioning groups. ${ }^{7}$ 
Following the reorganisation of NHS Primary Care, LAs took primary responsibility from the former primary care trusts (PCTs) for commissioning of the majority of public health services. However, the commissioning landscape for pharmacies is complex and variable across England, with different ways of working and subcontracting of commissioning responsibilities operating in some areas. ${ }^{7}$ Further, some services have been decommissioned ${ }^{8}$ in the face of resource challenges and lack of evidence of their effectiveness and cost-effectiveness. ${ }^{9}{ }^{10}$ In addition, while pharmacy public health services are well accepted by users and the general public is willing to use them, awareness of the services offered and how to access them remains limited, potentially contributing to low usage and consequential decommissioning. ${ }^{11-15}$

Localised commissioning, coupled with this continual flux, has resulted in a mosaic of services being offered through pharmacies across the country. Pharmacists may provide a range of private services in addition to those which are commissioned and publicly funded, ${ }^{16}$ such as blood pressure monitoring and private weight management services, but little is known about these on a national level. In addition, while most PCTs historically reported services commissioned to the Health and Social Care Information Centre (http://www.hscic.gov.uk) prior to the reorganisation of the NHS, some descriptor categories did not allow for individual services to be identified, further contributing to the lack of detailed information available. Since reorganisation, data reported publicly are very limited indeed. Local Pharmaceutical Committees are encouraged to provide data to a database maintained by the Pharmaceutical Services Negotiating Committee (PSNC) (http://www.psnc.org.uk), which is freely accessible, and many commissioners encourage pharmacists to submit data to a national database, such as PharmOutcomes (https://www.pharmoutcomes.org) and Service PACT (https://www.servicepact.co.uk). However, not all do so, for all services; thus, both sources of data are incomplete and neither is publicly available.

An analysis of the PSNC database in November 2015 suggested that a total of 558 public health services were commissioned by LAs from pharmacies across England. The most common services identified were: supervised administration of opioid substitutes to treat drug addiction, needle and syringe programmes (NSPs) and stop smoking services. ${ }^{17}$ However, this analysis relied on voluntary reporting to a database; thus, it was likely to be incomplete. More recently, in response to a proposal from the Department of Health to make significant funding cuts to the community pharmacy budget from December 2016, the PSNC commissioned a review of selected pharmacy services. Three public health services were included: supervised administration, NSP and emergency hormonal contraception (EHC). ${ }^{18}$ This review made use of both the PSNC and PharmOutcomes databases to estimate the volume and cost of delivering these services through community pharmacies in an attempt to assess their value to the NHS, society and users. However, pharmacy public health services are not limited to these three. A range of others are commissioned including smoking cessation, provision of free condoms, chlamydia screening and treatment, alcohol screening and brief advice, weight management, NHS Health Checks and vaccination services. ${ }^{17} \mathrm{~A}$ national picture of services is desirable to inform policy-makers and commissioners of both the overall extent of provision and variation across the country.

This study sought to obtain information about the extent of such provision through requests to LA commissioners under the Freedom of Information Act 2000, which provides public right of access to information held by public authorities.

\section{Aim}

This study aims to describe the extent of, and variation within, LA-commissioned pharmacy public health services across England.

\section{Methods}

We sent a freedom of information (FOI) request to all 152 LAs in England in July 2015, requesting details of any public health services commissioned from community pharmacies in their authority. The authorities that failed to respond, and those which directed us to their published Pharmaceutical Needs Assessment (PNA) documents, were sent a second, shorter request in February 2016 that focused on numbers of pharmacies commissioned and the number of service episodes completed. Some LAs failed to provide the relevant data after two requests and, in order to provide as complete a picture as possible, we approached the owners of PharmOutcomes (Pinnacle Health in partnership with Health Information Exchange) and Service PACT (Webstar Health) (two data packages supporting delivery of community pharmacy services) to supplement the data.

\section{Data handling and analysis}

We determined the number of different public health services commissioned, numbers of pharmacies commissioned and service usage for each LA. For non-responding LAs and where data were missing, we extracted data on services commissioned and the numbers of pharmacies commissioned, where available, from the PNA for each LA. To protect confidentiality, available usage data from the pharmacy data suppliers were added in aggregate form only and none is identifiable to a particular LA. The definitions we used to estimate usage were relevant to the nature of each service and are listed in table 1.

We calculated the total number of different public health services commissioned for England and for each LA. The numbers of community pharmacies in England and in each LA were obtained from the Local Government Authority website (http://lginform.local.gov.uk/ reports $/$ lgastandard? mod-metric $=3706 \&$ mod-period $=$ $1 \&$ mod-area=E92000001\&mod-group=AllLaInCountry_ England), enabling the proportion of pharmacies 


\begin{tabular}{|c|c|}
\hline Service & Definition of service use \\
\hline $\begin{array}{l}\text { Emergency hormonal } \\
\text { contraception }\end{array}$ & $\begin{array}{l}\text { Number of consultations (and } \\
\text { number of supplies if available) }\end{array}$ \\
\hline Chlamydia screening & $\begin{array}{l}\text { Number of screening kits provided } \\
\text { (and returned to laboratory if } \\
\text { available) }\end{array}$ \\
\hline Chlamydia treatment & Number of treatment episodes \\
\hline Free condoms & Number of registered users \\
\hline Supervised consumption & Number of individual supervisions \\
\hline $\begin{array}{l}\text { Needle and syringe } \\
\text { programmes }\end{array}$ & Number of needle packs \\
\hline Smoking cessation & $\begin{array}{l}\text { Number of registered users (plus } \\
\text { 4-week quitters if available) }\end{array}$ \\
\hline NHS Health Checks & Number of Health Checks \\
\hline Alcohol screening & Number of screens \\
\hline Weight management & Number of registered users \\
\hline $\begin{array}{l}\text { Healthy Start vitamin } \\
\text { supply }\end{array}$ & $\begin{array}{l}\text { Number of registered users and } \\
\text { number of supplies }\end{array}$ \\
\hline
\end{tabular}

providing services across England, and within each LA, to be calculated. Population health data relevant to the services provided were obtained from PHE (https://www. gov.uk/guidance/phe-data-and-analysis-tools) to enable us to compare distribution of services to proxy measures of health need. The correlations (Spearman's correlation coefficient) between datasets which we explored were: EHC provision against under 18 pregnancy rate, smoking support services against adult smoking rates, supervised consumption and NSP provision against deaths from substance use, alcohol screening against alcohol-related adult hospital admissions and NHS Health Checks against diabetes prevalence and under-75 cardiovascular mortality.

NHS England commissioned an influenza and pneumococcal vaccination service across all London boroughs in the winter of 2014/15 involving 1272 pharmacies. To calculate the number of pharmacies across England providing influenza vaccination, we combined these figures with data obtained from LAs outside London. However, as no usage data were available for the London service, it was not possible to determine national usage.

Approval by an NHS Research Ethics Committee was not necessary as no patients, users or carers were involved and no patient identifiable data were included in this project. Further, no approvals were required under research governance arrangements as no NHS organisations were included in the project.

\section{Results}

Response rate

The overall response rate was 146/152 (96.0\%): 127 usable responses to the first request and 17 to the second (two indicated that services were commissioned by third parties and provided no data). Four LAs indicated that services were commissioned by a neighbouring authority; thus, usable responses were obtained from 144 of the 148 commissioning authorities $(97.3 \%)$.

Usage data were not provided by 26 LAs. However, PharmOutcomes and Service PACT were able to supply data for at least one service for 14 of these, resulting in just 12/148 LAs (8\%) with no usage data for any service.

\section{Overview of commissioned services}

Information on the services commissioned was available for all 148 LAs, either from the FOI responses or from PNAs. A total of 833 services were reported as being commissioned, with those reported most frequently covering sexual health, services to people who use drugs (NSPs and supervised consumption) and smoking cessation (additional datafile 1). Relatively few authorities commissioned services relating to cardiovascular screening, lifestyle-related services or vaccinations. There were at least five services commissioned in $80 \%$ of all LAs, with a median of five services per LA (range 3-10).

Of the 833 services, 130 (15.5\%) were explicitly stated, or appeared, to be commissioned indirectly through third parties, such as via Drug and Alcohol Teams or integrated NHS trusts. This was most common for services to people who use drugs and smoking cessation, but some sexual health services were also externally commissioned, as were some small-scale services (figure 1). Data on the number of pharmacies providing commissioned services in each LA were $91 \%$ complete, with least data available for rarely commissioned services. table 2 shows the proportion of pharmacies commissioned in England as a whole for the range of services and also illustrates the variation across LAs. Significant variation across NHS Area teams was apparent for all major services (additional datafile 2). Fewer data were obtained on service use across the LAs (58\%) (table 2), but the available data do illustrate both the extent to which the services are accessed and the variation across LAs.

\section{Individual services}

Almost all LAs reported commissioning at least one pharmacy sexual health service (144; 97\%), with the most widespread service being EHC, being commissioned in $144 \mathrm{LAs}(97 \%)$ and provided by at least 47\% (5529) of the 11819 pharmacies registered in England at 29 November 2014. Chlamydia screening/treatment was available in $95(64 \%)$ LAs with at least $28 \%$ of pharmacies (3315) providing this service. There were five LAs where an oral contraception service was commissioned, and one commissioned a fitting service for long-acting reversible contraception across three pharmacies. Pregnancy testing was also mentioned as a service by several LAs, but it was more frequently incorporated into an integrated sexual health service.

Supervised consumption of opioid substitution therapy was the most frequently reported service for people who use drugs and was commissioned by all 148 LAs, provided by over half of all pharmacies in England (6186; 52\%). 
NSPs were commissioned from pharmacies in 145 (98\%) LAs, although the number of pharmacies providing this service was much lower than supervised consumption at $20 \%$ (2315).

Smoking cessation was by far the most frequently commissioned lifestyle modification service, in 133 (90\%) of LAs, and provided by 5660 pharmacies in England (48\%). Only 22 LAs (15\%) had commissioned an alcohol screening and brief intervention service and six (4\%) commissioned weight management services, while NHS Health Checks were commissioned by 48 (32\%) LAs.

Only 13 LAs outside London reported that they commissioned an influenza vaccination service, but five of these were specifically for staff employed by LAs. Assuming that all 33 London boroughs were commissioned to provide this service, a total of 46 LAs (31\%) had a commissioned service, involving 1531 pharmacies (in 44 of the 46 LAs). Two other LAs commissioned vaccination against hepatitis B. Among other smaller scale services, 10 LAs commissioned pharmacies to provide Healthy Start vitamins through the NHS voucher scheme, five commissioned testing for HIV, a further three commissioned screening services for blood-borne viruses (potentially covering both HIV and hepatitis B and C) and two commissioned directly observed treatment for tuberculosis.

\section{Comparison to population health data}

The variation in the proportion of pharmacies within LAs across England providing services to people who use drugs, EHC, smoking cessation, alcohol screening and NHS Health Checks showed poor correlations with relevant measures of population health in these LAs (table 3 and additional datafile 3). For example, 14 LAs did not report a commissioned pharmacy smoking cessation service, seven of which had adult smoking rates above the national average, while in 16 out of 61 other LAs (26\%) with above-average smoking rates, fewer than $50 \%$ of pharmacies provided a service. Only 48 LAs commissioned NHS Health Checks in pharmacies, $31(65 \%)$ of these were in areas with below-average diabetes prevalence and $29(60 \%)$ in areas with lower than average cardiovascular mortality rates. Of the 30 areas with the highest cardiovascular mortality rates, only six had commissioned pharmacies to provide NHS Health Checks, in contrast to 14 of the 30 LAs (47\%) with the lowest rates commissioning services. Only nine $(41 \%)$ of the 22 LAs commissioning alcohol screening from pharmacies had above-average rates of alcohol-related hospital stays.

\section{DISCUSSION}

The commissioning, subsequent provision and uptake of public health services in pharmacies is highly variable across England, with a wide range in number of services commissioned and in the proportion of pharmacies providing these within each LA. However, four services (EHC, smoking cessation support, supervised consumption of methadone or other medicines and NSPs) were

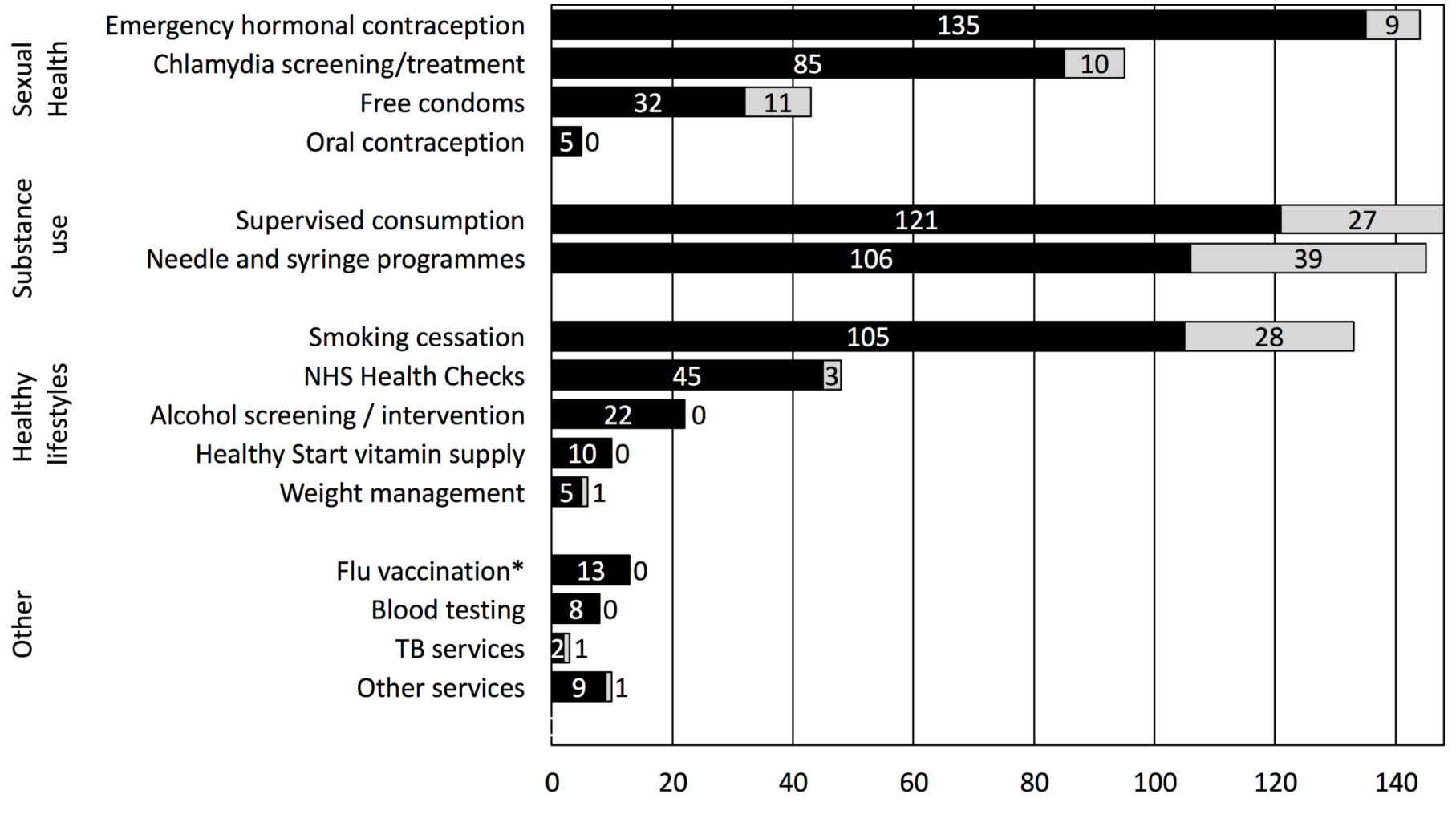

Number of LAs directly commissioning the service $\quad \square$ Number of LAs indirectly commissioning the service

Figure 1 Number of local authorities (LAs) commissioning each service directly or indirectly ( $n=148)\left({ }^{*}\right.$ Flu vaccination data excludes the service commissioned across London). NHS, National Health Service; TB, tuberculosis. 


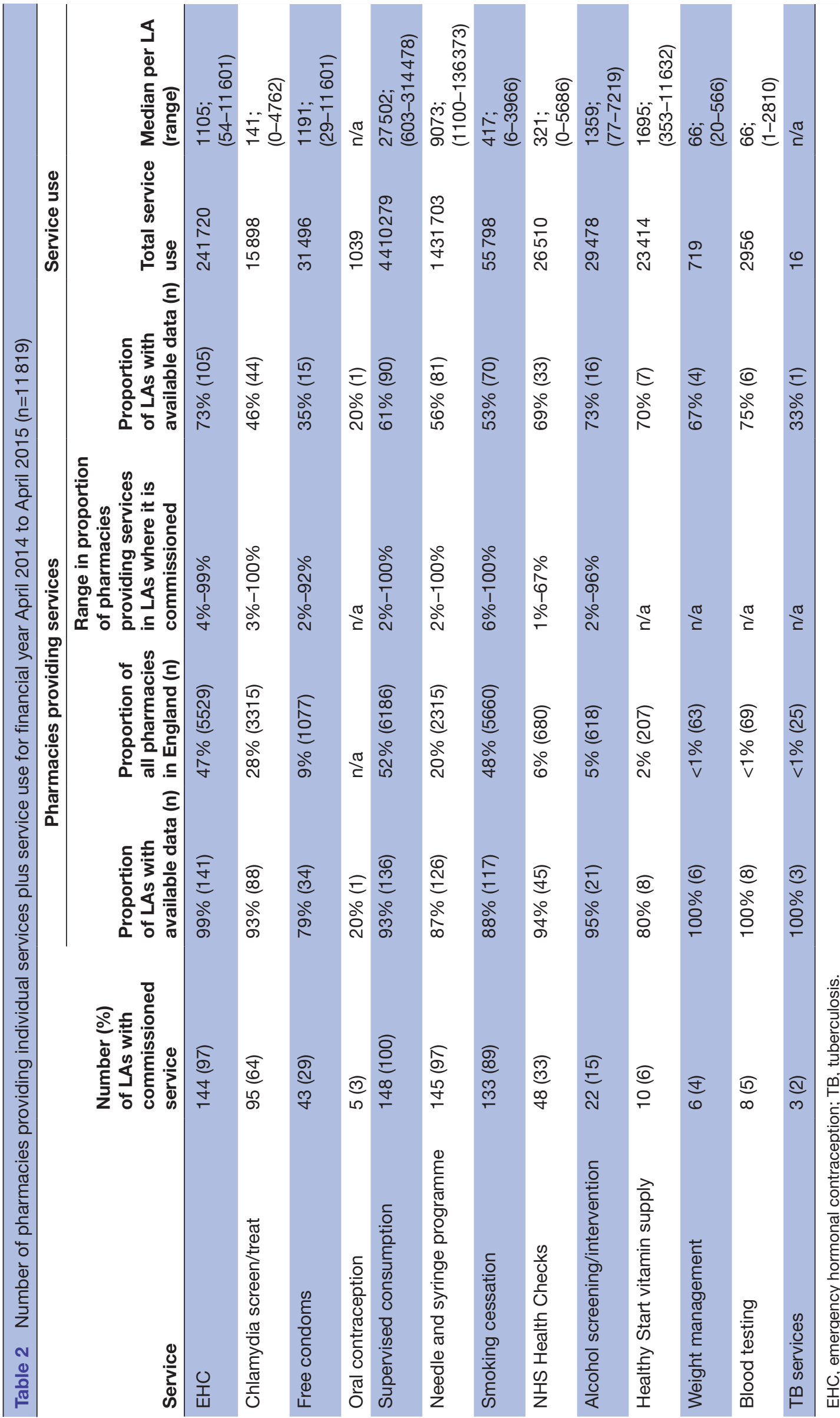

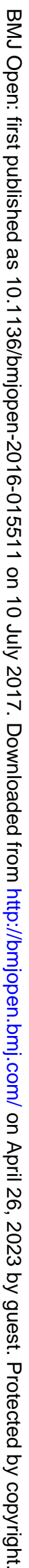


Table 3 Correlations between proportion of pharmacies commissioned to provide services and population health data for local authorities in England

\begin{tabular}{|c|c|c|c|c|}
\hline Pharmacy service & Health data & $\begin{array}{l}\text { Correlation } \\
\text { (Spearman's r) }\end{array}$ & $\mathbf{n}$ & p Value \\
\hline $\mathrm{EHC}$ & Under 18 pregnancy rate & 0.161 & 140 & 0.057 \\
\hline Supervised consumption & Deaths from substance use & 0.113 & 79 & 0.321 \\
\hline Needle and syringe programmes & Deaths from substance use & -0.055 & 77 & 0.633 \\
\hline NHS Health Checks & Under-75 cardiovascular mortality & -0.146 & 45 & 0.338 \\
\hline Alcohol screening/intervention & Hospital admissions related to alcohol in adults & 0.321 & 23 & 0.135 \\
\hline
\end{tabular}

EHC, emergency hormonal contraception; NHS, National Health Service.

all commissioned by at least $90 \%$ of LAs, with the first three being provided by at least $45 \%$ of all pharmacies in England. Supervised consumption and NSPs were the most frequently used service, with over 4.4 million individual supervisions and over 1.4 million needle packs supplied. There were over 200000 consultations with a pharmacist for supply of EHC, over 30000 received supplies of free condoms and almost 16000 received chlamydia screening kits provided by a pharmacy. Over 55000 people registered to stop smoking in a community pharmacy, almost 30000 were screened for alcohol use and over 26000 NHS Health Checks were carried out in pharmacies. However, commissioning of services did not appear to relate well to potential for benefit, estimated via local population health measures; indeed, for several services, the correlations obtained were negative.

This study provides the most comprehensive picture of public health services commissioned by English LAs from community pharmacies to date, covering the financial year 2014/15. A high response rate from the 148 commissioning LAs was achieved, some data being provided by $144(97.3 \%)$, with much missing data obtained from other sources; thus, the range of commissioned services across all LAs is complete, the number of pharmacies providing services is $90.2 \%$ complete (available for $751 / 833$ services) and data on service usage are $57.5 \%$ complete $(479 / 833$ services).

The data on services commissioned on behalf of LAs by other agencies may also be incomplete, although the FOI requests did seek information on indirectly commissioned services. The study also did not attempt to seek information on services commissioned by Clinical Commissioning Groups, services relating to medicines support provided under the national community pharmacy contractual framework or minor ailments services. The data requested covered the financial year 1 April 2014 to 31 March 2015, and we had to rely on the accuracy of the data provided in responses to FOI requests and available in PNAs, as it was not possible to corroborate these against other sources of data. The analysis assumes that services were provided throughout the year; thus, some data may contain inaccuracies, caused by the dynamic nature of commissioning and provision of services. Our study relates only to the particular commissioning structure used in England; thus, the findings are only of direct relevance to this setting and not generalisable to other countries. However, the need to include community pharmacy in strategic planning of services to meet local health needs is relevant to anywhere that they form a part of the public health service landscape.

The study did not take account of the provision of similar services by other agencies, so it is possible that local health needs were being met through other service providers. Relevant measures of population health were used to assess potential health need for individual services across LA areas, but these are recognised as a proxy measure. Also, the cross-sectional nature of the study and the corresponding data on health need mean that it is not possible to fully describe the relationship between the two. However, the intention of including these data is to illustrate the disconnect between the apparent health need and the provision of services through community pharmacies and not to suggest any causative relationship.

There are few published data on the commissioning or provision of these services. The most recent data available from the Health and Social Care Information Centre covered the period before the reorganisation of primary care in April 2013, and data were available for only three services in financial year 2012/13 (supervised consumption of opioid substitution therapy, NSPs and smoking cessation) and were also incomplete, being available for only 137 of the 153 commissioning trusts in England at the time. In this year, $51 \%$ of pharmacies provided supervised consumption, $20 \%$ provided NSPs and $55 \%$ provided smoking cessation services. There appears to be little change in these proportions in $2014 / 15$, being $52 \%, 20 \%$ and $48 \%$, respectively. Our data indicate that the report in November 2015, which estimated that supervised consumption was commissioned in 89 LAs, NSPs were commissioned in 86, stop smoking services were commissioned in 88 , EHC was commissioned in 71 and chlamydia screening was commissioned in $47,{ }^{17}$ was a significant underestimate. More recently, estimates 
based on data provided to PSNC indicated that supervised administration was commissioned in 130 LAs, NSPs were in 111 and EHC was in $131 .{ }^{18}$ This too appears to underestimate the extent of commissioning; however, the estimate of 6158 pharmacies providing supervised consumption given in this report is in line with our findings (6186). The variation found here is in contrast to the nationally commissioned influenza vaccination service introduced in 2015/16, which involved a total of 7195 pharmacies, which provided 595467 vaccinations, with the proportion of pharmacies providing the service across NHS area teams ranging from $53 \%$ to $76 \% .^{19}$

Community pharmacy has long been championed as a potential setting for the delivery of public health services to significant sectors of the communities in which they are located, primarily because of their accessibility. While a few key services are widely commissioned from community pharmacies by LAs and are extensively used, there is significant variation in the distribution of these services, which appears to be poorly correlated to potential need. Other services such as NHS Health Checks and screening for risky alcohol use, for which there is less evidence of benefit, are much less frequently commissioned but again are not concentrated in areas where the local community is most likely to benefit. The reasons for the variation we have found are not clear, from our work or the available literature, but do warrant investigation. There is a need to re-visit the benefits of local commissioning of these services, which may be influenced by a range of factors other than identified need. The poor, sometimes negative, correlations we found suggest that commissioners should consider more strategically how community pharmacies could most usefully contribute to addressing local public health issues. Regional or national approaches to commissioning, designed to allow flexibility in the extent of service provision based on differing needs, but using common service specification structures and data templates, could facilitate better large-scale data gathering. As our study shows, this is not the case with the current situation.

Acknowledgements We are grateful to the LAs that diligently and comprehensively responded to our initial and subsequent requests. We acknowledge the assistance of Alastair Thomas in distributing the initial FOl request and recording data and are extremely grateful to Gary Warner (Pharm0utcomes) and Gian Celino (Service PACT) for providing aggregated service usage data.

Contributors AJM and JK conceived and designed the study. JK and NJG performed the analysis of the data. AJM, JK and NJG interpreted the results. AJM and JK drafted the manuscript and all authors contributed with critical revisions to the contents of the manuscript. The final version of the manuscript was approved by all authors.

Funding This work was funded by Medway School of Pharmacy and a grant from Public Health England (PHE). PHE had no influence on the design, analysis or reporting of the study and the views expressed in this paper are those of the authors and do not necessarily reflect those of PHE.

Competing interests AJM has received payments in respect of locum pharmacist work from one community pharmacy owning company. In the previous 3 years, NJG has received research funding for other projects from Pharmacy Research UK, Greater Manchester Local Pharmaceutical Committee (formerly Community Pharmacy Greater Manchester), Webstar Health Limited and an independent pharmacy company, and payment from PHE via the University of Kent to participate in this project, all of which organisations might have an interest in the submitted work. NJG's spouse has financial relationships that may be relevant to the submitted work. AJM provided advice to NICE on the scope for a guideline relating to community pharmacy's promotion of health and well-being and is an Expert Member of the NICE Public Health Advisory Committee that is developing this guideline. AJM and NJG are registered pharmacists in Great Britain. No other relationships or activities that relate to the submitted work exist.

Provenance and peer review Not commissioned; externally peer reviewed.

Data sharing statement Data on the numbers of pharmacies in each Local Authority commissioned to provide the individual services is available from the corresponding author.

Open Access This is an Open Access article distributed in accordance with the Creative Commons Attribution Non Commercial (CC BY-NC 4.0) license, which permits others to distribute, remix, adapt, build upon this work non-commercially, and license their derivative works on different terms, provided the original work is properly cited and the use is non-commercial. See: http://creativecommons.org/ licenses/by-nc/4.0/

(c) Article author(s) (or their employer(s) unless otherwise stated in the text of the article) 2017. All rights reserved. No commercial use is permitted unless otherwise expressly granted.

\section{REFERENCES}

1. Clucas K. Pharmacy: the report of a committee of inquiry appointed by the Nuffield Foundation. London, 1986.

2. Department of Health. Pharmacy in England: building on strengths delivering the future. London: The Stationery Office, 2008.

3. Royal Pharmaceutical Society of Great Britain. Pharmacy in a new age: developing strategy for the future of pharmacy. London, 1995.

4. Pharmaceutical Services Negotiating Committee. The Vision for NHS Community Pharmacies: the path to improved patient care. London, 2013.

5. Smith J, Picton C, Dayan M. Now or never : shaping pharmacy for the future. London, 2013.

6. Todd A, Copeland A, Husband A, et al. The positive pharmacy care law: an area-level analysis of the relationship between community pharmacy distribution, urbanity and social deprivation in England. BMJ Open 2014;4:e005764.

7. Smith J, Picton C, Dayan M. Now more than ever: why pharmacy needs to act. London, 2014.

8. Gray NJ, Wilson SE, Cook PA, et al. Understanding and optimising an identification / brief advice (IBA) service about alcohol in the community pharmacy setting. Final Report. Liverpool, 2012.

9. Brown TJ, Todd A, O?Malley CL, et al. Community pharmacy interventions for public health priorities: a systematic review of community pharmacy-delivered smoking, alcohol and weight management interventions. Public Health Res 2016;4:1-162.

10. Newton J. Pharmacy \& Public Health Forum. Consolidating and developing the evidence base and research for community pharmacy's contribution to public health: a progress report from Task Group 3 of the Pharmacy and Public Health Forum About Public Health England. London, 2013.

11. Agomo CO. The role of community pharmacists in public health: a scoping review of the literature. J Pharm Health Serv Res 2012;3:25-33.

12. Krska J, Morecroft CW. Views of the general public on the role of pharmacy in public health. J Pharm Health Serv Res 2010;1:33-8.

13. Taylor J, Krska J, Mackridge A. A community pharmacy-based cardiovascular screening service: views of service users and the public. Int J Pharm Pract 2012;20:277-84.

14. Saramunee K, Krska J, Mackridge A, et al. How to enhance public health service utilization in community pharmacy?: general public and health providers' perspectives. Res Social Adm Pharm 2014;10:272-84.

15. Saramunee K, Krska J, Mackridge A, et al. General public's views on pharmacy public health services: current situation and opportunities in the future. Public Health 2015;129:705-15.

16. Dewsbury C, Rodgers R, Krska J. Views of English pharmacists on providing public health services. Pharmacy 2015;3:154-68.

17. Robinson $\mathrm{S}$. How cuts to public health funding could affect pharmacy. Pharmaceutical Journal 2015;295.

18. Cooper PW. The value of community pharmacy - detailed report PSNC 2015 http://psnc.org.uk/wp-content/uploads/2016/09/The-value-ofcommunity-pharmacy-detailed-report.pdf (accessed 14 Nov 2016).

19. Connelly C. Leading providers of influenza vaccinations in English Pharmacies. Pharm J 2016;279:216-7. 